\title{
Characterizing Colonization Patterns of Clavibacter michiganensis During Infection of Tolerant Wild Solanum Species
}

\author{
F. Christopher Peritore-Galve, ${ }^{1}$ Christine Miller, ${ }^{1,2}$ and Christine D. Smart ${ }^{1, \dagger}$ \\ ${ }^{1}$ Plant Pathology and Plant-Microbe Biology Section, School of Integrative Plant Science, Cornell University, Geneva, NY 14456 \\ 2 Department of Plant and Microbial Biology, North Carolina State University, Raleigh, NC 27606 \\ Accepted for publication 12 November 2019.
}

\begin{abstract}
Clavibacter michiganensis is the Gram-positive causal agent of bacterial canker of tomato, an economically devastating disease with a worldwide distribution. C. michiganensis colonizes the xylem, leading to unilateral wilt, stem canker, and plant death. C. michiganensis can also infect developing tomato fruit through splash dispersal, forming exterior bird's eye lesions. There are no documented sources of qualitative resistance in Solanum spp.; however, quantitative trait loci conferring tolerance in Solanum arcanum and Solanum habrochaites have been identified. Mechanisms of tolerance and C. michiganensis colonization patterns in wild tomato species remain poorly understood. This study describes differences in symptom development and colonization patterns of the wild type (WT) and a hypervirulent bacterial expansin knockout ( $\triangle$ CmEXLX2) in wild and cultivated tomato genotypes. Overall, WT and $\triangle \mathrm{CmEXLX} 2$ cause less severe symptoms in wild tomato species and are impeded in spread and colonization of the vascular system.
\end{abstract}

ABSTRACT
Laser scanning confocal microscopy and scanning electron microscopy were used to observe preferential colonization of protoxylem vessels and reduced intravascular spread in wild tomatoes. Differences in C. michiganensis in vitro growth and aggregation were determined in xylem sap, which may suggest that responses to pathogen colonization are occurring, leading to reduced colonization density in wild tomato species. Finally, wild tomato fruit was determined to be susceptible to $C$. michiganensis through in vivo inoculations and assessing lesion numbers and size. Fruit symptom severity was in some cases unrelated to severity of symptoms during vascular infection, suggesting different mechanisms for colonization of different tissues.

Keywords: bacteriology, bacterial canker, Clavibacter michiganensis, Gram-positive, plant tolerance, tomato, wild tomatoes, xylem
Bacterial canker is one of the most economically damaging diseases affecting tomato production worldwide (de León et al. 2011; Nandi et al. 2018; Sen et al. 2015). Clavibacter michiganensis, formerly Clavibacter michiganensis subsp. michiganensis, is the Gram-positive causal agent of bacterial canker within the genus Clavibacter, which has undergone reclassification from subspecies to species rank (Li et al. 2018; Thapa et al. 2019). C. michiganensis systemically colonizes the tomato vascular system, leading to unilateral wilt, marginal leaf necrosis, stem cankers, and plant death (Chalupowicz et al. 2012; Sen et al. 2015). Splash dispersal of $C$. michiganensis can infect the exterior of developing tomato fruit, producing bird's eye lesions that affect fruit quality and can lead to seed contamination (Medina-Mora et al. 2001; Tancos et al. 2013). Systemic spread of C. michiganensis in the xylem makes it difficult to control with conventional products, and there is no commercially available resistance in tomato (de León et al. 2011; Sen et al. 2015). Quantitative trait loci (QTL) from wild tomato species that confer tolerance have been identified; however, the mechanisms of tolerance to $C$. michiganensis are poorly understood (Sen et al. 2015). Studying $C$. michiganensis colonization of tomatoes with varying susceptibility is a step toward deciphering how wild tomato species tolerate $C$. michiganensis infection.

\section{${ }^{\dagger}$ Corresponding author: C. D. Smart; cds14@cornell.edu}

Funding: This work was supported by National Institute of Food and Agriculture, U.S. Department of Agriculture, Federal Capacity Funds project 1014252 and National Institute of Food and Agriculture, U.S. Department of Agriculture grant 2016-67032-25009 for summer undergraduate research.

*The $e$-Xtra logo stands for "electronic extra" and indicates that seven supplementary figures are published online.

The author(s) declare no conflict of interest.

(C) 2020 The American Phytopathological Society
Wild Solanum spp. harbor genetic diversity that can be exploited as sources for disease resistance. Several species, including $S$. arcanum, S. chilense, S. glandulosum, S. habrochaites, S. minutum, $S$. parviflorum, $S$. peruvianum, and $S$. pimpenellifolium, have demonstrated moderate to high tolerance to $C$. michiganensis (Francis et al. 2001; Kabelka et al. 2002; Lara-Ávila et al. 2012; Sen et al. 2012, 2015; Sotirova et al. 1999; van Heusden et al. 1999; Vulkova and Sotirova 1993). Genetic studies of $S$. arcanum LA2157 (formerly Lycopersicon peruvianum) identified additive and codominant QTL on different chromosomes of interspecific crosses and an $\mathrm{F}_{2}$ population (Sotirova et al. 1999; van Heusden et al. 1999). Fine mapping of S. habrochaites LA407 (formerly Lycopersicon hirsutum) identified two QTL with additive tolerance on chromosomes 2 and 5 that contribute to vascular development, morphology, and $\mathrm{H}_{2} \mathrm{O}_{2}$ defense responses in the xylem (Coaker and Francis 2004; Coaker et al. 2002, 2004; Francis et al. 2001). Despite low symptom development in wild Solanum spp., $C$. michiganensis is able to systemically colonize the vascular system to high densities $\left(10^{4}\right.$ to $10^{8} \mathrm{CFU}$ per $1 \mathrm{mg}$ of tissue) (Francis et al. 2001; Sen et al. 2012). To the best of our knowledge, there have been no published studies investigating wild tomato fruit susceptibility to $C$. michiganensis. The role of C. michiganensis colonization, spread, and plant response in the development of bacterial canker symptoms remains uncharacterized in these tolerant plant species.

C. michiganensis interacts with plant host tissues in a complex manner that is poorly understood (Chalupowicz et al. 2017; Nandi et al. 2018). Genomic analyses have revealed a pathogenicity island and two plasmids necessary for full virulence in the reference strain, NCPPB382 (Dreier et al. 1997; Gartemann et al. 2008; Jahr et al. 2000; Meletzus et al. 1993). These mobile elements contain multiple copies of genes encoding secreted serine proteases, carbohydrate active enzymes, expansins, and tomatinase (Gartemann et al. 2008). Genetic studies have demonstrated that $C$. michiganensis strains have variable gene and plasmid contents that contribute to virulence (Tancos et al. 2015; Thapa et al. 2017). In a previous study, a 
hypervirulent $C$. michiganensis isolate was generated by knocking out a single-copy expansin gene encoding a secreted expansin-like protein (CmEXLX2) (Tancos et al. 2018). The role of secreted C. michiganensis expansin proteins during virulence and the mechanisms underlying hypervirulence remain unclear, but the hypervirulent strain $\triangle$ CmEXLX2 can be used as a tool to further our understanding of host-C. michiganensis interactions.

C. michiganensis poses a major threat to tomato producers, and epidemics often begin through seedling transplants harboring latent infections (de León et al. 2011). The goals of this study were to understand differences in $C$. michiganensis colonization and host symptom development between susceptible domesticated tomato and tolerant wild tomato species. Results from this study can begin to delineate the complex relationship between $C$. michiganensis and the host tissues that it colonizes.

\section{MATERIALS AND METHODS}

Disease severity assays. Wild tomato seeds ( $S$. habrochaites LA2128, S. arcanum LA2157, and S. arcanum LA2172) were washed in a $50 \%$ bleach solution for $15 \mathrm{~min}$, rinsed with sterile deionized water, and then, germinated on wet sterile Whatman filter paper (Fisher Scientific) in petri dishes for 8 to 10 days. Seedlings were then transferred to a Farfard professional formula growing mix in 72-cell flats (Sun Gro Horticulture), and Solanum lycopersicum 'Mt. Fresh Plus' seeds were started in the mix. When seedlings had two true leaves, they were transferred to 4-inch pots and supplemented with Osmocote slow release fertilizer (Scotts Miracle-Gro Co.). Plants were grown with a 16-h light/8-h dark photoperiod in an environmentally controlled greenhouse.

To characterize plant susceptibility to $C$. michiganensis, seedlings of S. lycopersicum 'Mt. Fresh', S. habrochaites LA2128, $S$. arcanum LA2157, and $S$. arcanum LA2172 between the twoand three-true leaf stage were inoculated with wild-type (WT) C. michiganensis 0317 (hereafter WT) and C. michiganensis $0317 \Delta \mathrm{CmEXLX} 2$ (hereafter $\triangle \mathrm{CmEXLX} 2$ ) cultured in LuriaBertani (LB) medium and LB supplemented with gentamicin $(40 \mu \mathrm{g} / \mathrm{ml})$, respectively, for $18 \mathrm{~h}$ with shaking at $160 \mathrm{rpm}$ at $28^{\circ} \mathrm{C}$ (Tancos et al. 2018). Bacterial suspensions were adjusted to optical density $600\left(\mathrm{OD}_{600}\right)=0.8$ through centrifugation for $12 \mathrm{~min}$ at 4,000 rpm, washing the pellet with sterile water, and resuspending in sterile water (Tancos et al. 2018). Tomato seedlings ( $n=15$ per genotype) were arranged in three randomized blocks with two replicates of each plant genotype/C. michiganensis strain combination per block in the greenhouse. One mock-inoculated plant per genotype was included in each block as a control.

Plants were inoculated by dipping sterilized scissors into bacterial suspension and clipping the cotyledons (Tancos et al. 2018). Plants were assessed daily for leaflet wilting and stem canker formation. Observations continued until 21 days postinoculation (dpi). Disease severity was quantified by dividing the number of leaflets wilting each day by the total number of leaflets present on the oldest seven leaves at 21 dpi (Balaji et al. 2008; Chalupowicz et al. 2012; Tancos et al. 2018). The mean area under the disease progress curve (AUDPC) was calculated from disease severity measurements (Tancos et al. 2018). Differences in AUDPC were analyzed using a mixed effects model with strain, genotype, block, and the interaction between strain and genotype as explanatory variables and a random effect to account for block effects using packages lme4, pbkrtest, emmeans, and lsmeans in R v. 3.3.2 (Bates et al. 2015; Halekoh and Højsgaard 2014; Lenth 2016). Significant differences between treatment groups were tested using Tukey's honestly significant difference test (HSD) test $(P<0.05)$. The experiment was performed three times.

Bacterial movement and in planta growth. To characterize spread and colonization density of $C$. michiganensis in the plant vascular system, $0.5-\mathrm{cm}$ sections of tomato stem tissue at the inoculation site $(0 \mathrm{~cm}), 5 \mathrm{~cm}$ above, and $10 \mathrm{~cm}$ above were harvested at $21 \mathrm{dpi}(n=3$ per genotype inoculated with WT or $\Delta$ CmEXLX2).
Tissues were weighed and homogenized in $1 \mathrm{ml}$ of sterile water using a sterile 5-mm stainless steel grinding bead (Qiagen) in a 2-ml Eppendorf tube using a TissueLyser (Retsch) at $30 \mathrm{~Hz}$ for $4 \mathrm{~min}$. One hundred microliters of the homogenate was transferred to a 96well Falcon tissue culture plate (Corning Inc), and $10 \mu \mathrm{l}$ were serial diluted into $90 \mu \mathrm{l}$ of sterile water for seven dilutions. Ten microliters of each dilution was spot inoculated onto D2ANX or LB plates amended with gentamycin $(40 \mu \mathrm{g} / \mathrm{ml})$ four times and incubated for 2 to 3 days at $28^{\circ} \mathrm{C}$. Numbers of $\mathrm{CFU}$ were quantified at the dilution where there was between 20 and $200 \mathrm{CFU}$, and the average of four technical replicates per biological replicate was used for the final analysis. CFU counts were $\log _{10}$ transformed, and data from three individual experiments were analyzed separately and then pooled for the final analysis $(n=9$ biological replicates of a host genotype inoculated with WT or $\triangle \mathrm{CmEXLX} 2$ at 0,5 , and $10 \mathrm{~cm}$ from the inoculation site). A mixed effects model was generated with $\log _{10}$ CFU milligram ${ }^{-1}$ of tissue as the response and distance by genotype by strain as the response with random effect terms accounting for variation between experiments, blocks within experiments, and variability within a single plant. The analysis was performed using packages lme4, pbkrtest, emmeans, and lmerTest in R v. 3.3.2 (Bates et al. 2015; Halekoh and Højsgaard 2014; Lenth 2016). Significant differences between groups were tested using Tukey's HSD test $(P<0.05)$.

In vitro growth and attachment of $C$. michiganensis in xylem sap. Xylem sap for in vitro analyses was extracted from plants between the six- and eight-leaf stage by heavy watering the night before and at dawn, cutting the stem at an angle approximately $1 \mathrm{~cm}$ above the cotyledons; then, we tilted the pots to allow sap to drain into 15-ml conical tubes for $1 \mathrm{~h}$. Sap was transferred into tubes on ice every $15 \mathrm{~min}$. Sap from at least six plants was pooled and filtered through $0.2-\mu \mathrm{m}$ polyethersulfone syringe filters (Corning Inc.) and stored at $-20^{\circ} \mathrm{C}$.

To measure bacterial growth in xylem sap, WT and $\triangle \mathrm{CmEXLX} 2$ were grown as described above and resuspended to $\mathrm{OD}_{600}=0.1$ in sterile water. Five microliters of three individual WT and $\triangle$ CmEXLX2 cultures were inoculated into 12 wells (4 wells per bacterial culture) of a 96-well Falcon tissue culture plate containing $195 \mu \mathrm{l}$ of sterile tomato sap. The plate was incubated at $28^{\circ} \mathrm{C}$ with shaking in a BioTek microplate reader. OD was measured at $590 \mathrm{~nm}$ every $2 \mathrm{~h}$ for $48 \mathrm{~h}$ (Tancos et al. 2018). OD for bacterial growth samples was normalized to mock (sterile water)-inoculated sap from each genotype. Area under the growth curve was calculated for each sample, and statistical analyses were performed using a mixed effects model including bacterial cultures as random effects using packages lme4, pbkrtest, and emmeans in R v. 3.3.2 (Bates et al. 2015; Halekoh and Højsgaard 2014; Lenth 2016). Significant differences between treatment groups were tested using Tukey's HSD test $(P<0.05)$. The experiment was performed twice.

To measure $C$. michiganensis attachment in vitro, WT and $\triangle \mathrm{CmEXLX2}$ were grown as described above and adjusted to $\mathrm{OD}_{600 \mathrm{~nm}}=0.8$. Five microliters of three individual cultures of WT and $\triangle \mathrm{CmEXLX} 2$ were inoculated to 12 wells ( 4 wells per bacterial culture) of a 96-well Falcon tissue culture plate containing $95 \mu \mathrm{l}$ of filter-sterilized tomato sap. Plates were briefly shaken and statically incubated at $28^{\circ} \mathrm{C}$ for 5 days (Chalupowicz et al. 2012; Tancos et al. 2018; Tran et al. 2016). After incubation, the supernatant was gently removed through pipetting, and wells were washed twice with $150 \mu \mathrm{l}$ of sterile water. The samples were fixed at $60^{\circ} \mathrm{C}$ for $1 \mathrm{~h}$, and surface-attached bacteria were stained with $25 \mu \mathrm{l}$ of $0.1 \%$ crystal violet for $25 \mathrm{~min}$ at room temperature. The plate was washed twice with $200 \mu \mathrm{l}$ of sterile water and gently blotted on paper towels (Davey and O'toole 2000; Tancos et al. 2018; Tran et al. 2016). Crystal violet was solubilized by adding $100 \mu \mathrm{l}$ of $30 \%$ acetic acid followed by brief agitation and quantifying the absorbance at $590 \mathrm{~nm}$ using the BioTek microplate reader (Davey and O'toole 2000; Tancos et al. 2018; Tran et al. 2016). Statistical analyses were performed using a mixed effects model including bacterial cultures 
as random effects using packages lme4, pbkrtest, and emmeans in $\mathrm{R}$ v. 3.3.2 (Bates et al. 2015; Halekoh and Højsgaard 2014; Lenth 2016). Significant differences between treatment groups were tested using Tukey's HSD test $(P<0.05)$. The experiment was performed twice.

Lateral C. michiganensis spread in primary vascular bundles. Three seedlings of each of the four host genotypes were grown as described above. Strains of WT and $\triangle$ CmEXLX2 with stable plasmids expressing eGFP were cultured as described in LB amended with kanamycin $(100 \mu \mathrm{g} / \mathrm{ml}$; Fisher Scientific) or kanamycin and gentamicin $(40 \mu \mathrm{g} / \mathrm{ml})$, respectively (Tancos et al. 2018). Cultures were adjusted to $\mathrm{OD}_{600}=0.8$ and cotyledon clip inoculated into plants at the two- to three-true leaf stage $(n=3$ per genotype per strain). Mock-inoculated plants were included as controls.

To determine the number of xylem vessels colonized by C. michiganensis, transverse stem sections were excised $1 \mathrm{~cm}$ above the inoculation site at $21 \mathrm{dpi}$. Primary vascular bundles were imaged using scanning laser confocal microscopy (Olympus BX61; Fluoview FV-300; Olympus Corp.) using argon (488-nm) and green helium neon (543-nm) lasers to excite eGFP-expressing bacteria and induce plant autofluorescence, respectively (Chalupowicz et al. 2012; Tancos et al. 2013, 2018). The number of infected and noninfected protoxylem and metaxylem vessels in primary vascular bundles was quantified for each of three plants for each genotype/ strain combination. Mock-inoculated plants were included as controls for plant autofluorescence. In total, 332 fields of view were imaged for quantification. Comparisons of percentage of infected xylem vessels were calculated with analysis of variance (ANOVA) and Tukey's HSD test $(P<0.05)$ using packages agricolae and emmeans in R v 3.3.2 (Lenth 2016; Mendiburu 2015).

To visualize bacterial colonization of individual proto- and metaxylem vessels using scanning electron microscopy (SEM), transverse and longitudinal stem sections $1 \mathrm{~cm}$ above the inoculation site were excised 12 dpi from mock- and WT-inoculated plants $(n=2$ per genotype). Tissue sections were fixed in $3 \%$ glutaraldehyde in sterile phosphate-buffered saline followed by application of vacuum pressure to remove air from samples. Tissues were washed four times with Sørensen's phosphate buffer (pH 7.2) and dehydrated using an ethanol series. Solvents were removed through critical point drying (030 Critical point dryer; BAL-TEC), and samples were mounted on aluminum stubs and sputter coated twice with gold (EMS500x; Electron Microscopy Sciences). Images were captured using a scanning electron microscope (S-530 SEM; Hitachi) equipped with a digital camera. Two hundred eight fields of view were imaged in total.

Fruit disease severity assays. To assess susceptibility of wild tomato fruit to $C$. michiganensis, 6-week-old plants were transferred to 3-gallon pots and fertilized with Osmocote slow release fertilizer. Eight-week-old S. lycopersicum 'Mt. Fresh' plants were self-pollenated using a vibrating pollinator wand (VegiBee). $S$. habrochaites LA2128 and both $S$. arcanum genotypes were manually pollinated. C. michiganensis strains were cultured and adjusted to $\mathrm{OD}_{600}=0.8$ as described above. Water, WT, and $\triangle$ CmEXLX2 were brush inoculated onto fruit between 7 and 9 days postanthesis (dpa; $n=10$ per treatment) of four plants of each genotype. To prevent cross-contamination of treatments, fruit clusters on the same plant were required to be a minimum of $25 \mathrm{~cm}$ apart. Fruits were harvested $15 \mathrm{dpi}$, and the diameter and number of lesions per fruit were measured. To measure fruit lesion size, images of each fruit were taken using an Olympus SZX18 dissecting microscope (Olympus Corp.). Lesion diameter and diameter of the lesion's necrotic center were measured using Olympus cellSens software (Olympus Corp.; $n=100$ per treatment). Comparisons of number of lesions per fruit and differences in lesion size were calculated using ANOVA and using Tukey's HSD test $(P<0.05)$ with packages agricolae, emmeans, and lsmeans in R v 3.3.2 (Lenth 2016; Mendiburu 2015). The fruit susceptibility experiment was performed twice for a total of 20 fruit per host genotype/C. michiganensis strain combination.

\section{RESULTS}

Wild tomato species express fewer bacterial canker symptoms. Host susceptibility and movement of C. michiganensis in the vascular system were assessed by inoculating seedlings of cultivated and wild tomatoes with $C$. michiganensis. Wild tomato seedlings inoculated with WT and $\triangle$ CmEXLX2 developed little to no leaflet wilt in comparison with S. lycopersicum 'Mt. Fresh' $(P<$ 0.01 ) (Supplementary Fig. S1). Leaflet wilt appeared as early as 7 and 10 dpi in S. lycopersicum 'Mt. Fresh' plants inoculated with $\triangle \mathrm{CmEXLX} 2$ and WT, respectively. On average, wilt appeared on $S$. arcanum LA2172 plants inoculated with $\triangle \mathrm{CmEXLX} 2$ around 12 dpi. Wilt symptoms of $S$. habrochaites LA2128 inoculated with both strains were delayed until $17 \mathrm{dpi}$. $\triangle \mathrm{CmEXLX} 2$ was hypervirulent in seedlings of $S$. lycopersicum 'Mt. Fresh' as well as $S$. arcanum LA2172 but not in S. habrochaites LA2128 and S. arcanum LA2157 (Fig. 1A and C).

Differences in bacterial canker symptoms were observed between tomato genotypes. Severely affected leaflets of $S$. arcanum LA2172 wilted without marginal leaf necrosis, differing from typical wilt symptoms of bacterial canker in S. lycopersicum 'Mt. Fresh' (Fig. 1C and Supplementary Fig. S2). S. habrochaites LA2128 leaflets would partially wilt before the development of chlorosis followed by marginal leaf necrosis (Supplementary Fig. S2). Both C. michiganensis strains produced typical stem cankers at the inoculation site between 9 and 12 dpi in S. lycopersicum 'Mt. Fresh' (Supplementary Fig. S2). Only $\triangle \mathrm{CmEXLX} 2$ was able to form stem cankers at the inoculation site of $S$. arcanum LA2172. No cankers were present in S. habrochaites LA2128 and $S$. arcanum LA2157 plants inoculated with either C. michiganensis strain (Supplementary Fig. S2).

C. michiganensis spread and colonization are reduced in wild tomatoes. The ability for $C$. michiganensis to spread and densely colonize the vascular system is critical for systemic infection and symptom development. We quantified WT and $\triangle \mathrm{CmEXLX2}$ colonization density of plant stem sections $21 \mathrm{dpi}$ at the inoculation site (0), 5 , and $10 \mathrm{~cm}$ above. WT C. michiganensis was able to colonize to similarly high densities ( $10^{9} \mathrm{CFU}$ per $1 \mathrm{~g}$ of tissue) at the inoculation sites of S. lycopersicum 'Mt. Fresh' and S. arcanum LA2172 but not of S. habrochaites LA2128 and S. arcanum LA2157 (Fig. 1B and Supplementary Fig. S3). WT C. michiganensis populations were reduced at 5 and $10 \mathrm{~cm}$ above the inoculation site of all wild tomatoes (Fig. 1B and Supplementary Fig. S3). $\triangle$ CmEXLX2 only colonized at significantly higher densities relative to WT $(P<0.05)$ at varying distances in $S$. arcanum accessions (Fig. 1B and Supplementary Fig. S3). Populations of $\triangle \mathrm{CmEXLX2}$ in wild tomatoes were also reduced as the distance from the inoculation site increased. Both WT and $\triangle \mathrm{CmEXLX} 2$ populations were lowest in the asymptomatic accession $S$. arcanum LA2157, but $\triangle$ CmEXLX2 colonization of $S$. arcanum LA2172 was almost equal to $\triangle \mathrm{CmEXLX} 2$ titers in $S$. lycopersicum 'Mt. Fresh', suggesting that $\Delta$ CmEXLX2 hypervirulence is specific to certain plant genotypes within a species (Fig. 1A).

Composition of tomato xylem sap affects growth and biofilm formation. The xylem sap composition of wild tomatoes could be suboptimal for $C$. michiganensis growth, therefore reducing in planta growth and symptom development. We extracted sap from the four tomato genotypes and measured in vitro growth rates of WT and $\triangle \mathrm{CmEXLX} 2$ for $48 \mathrm{~h}$. There were no differences in growth rates between WT and $\triangle \mathrm{CmEXLX} 2$ when cultured in sap from each of the four genotypes (Fig. 2). C. michiganensis grew to the highest density in sap from the most susceptible genotype, S. lycopersicum 'Mt. Fresh', and to the second highest density in sap from the asymptomatic genotype $S$. arcanum LA2157 (Fig. 2). C. michiganensis grew to lowest densities in sap from $S$. habrochaites LA2128. 
Using the crystal violet attachment assay, we determined that WT C. michiganensis was able to aggregate to similar levels in sap from $S$. lycopersicum 'Mt. Fresh' and $S$. arcanum genotypes (Supplementary Fig. S4). Aggregation was severely reduced in sap from $S$. habrochaites LA2128, perhaps because of lower in vitro growth (Fig. 2 and Supplementary Fig. S4). $\triangle$ CmEXLX2 formed fewer aggregates in sap from both $S$. arcanum genotypes in comparison with WT but was only significantly lower in sap from S. arcanum LA2157 $(P=0.0058)$.

$C$. michiganensis is impeded in lateral spread in wild tomato vascular bundles. The spread of $C$. michiganensis between xylem vessels of wild tomato species was studied by inoculating plants with eGFP-expressing WT and $\triangle$ CmEXLX2 strains and quantifying colonized proto- and metaxylem vessels in primary vascular bundles $21 \mathrm{dpi}$ using laser scanning confocal microscopy (Supplementary Fig. S5). WT and $\triangle$ CmEXLX2 colonized similar proportions of protoxylem vessels in all genotypes tested (Table 1). C. michiganensis was present in $>50 \%$ of protoxylem vessels in primary vascular bundles of all four plant genotypes (Table 1). Spread to metaxylem vessels by C. michiganensis was highest in the most susceptible genotypes (S. lycopersicum 'Mt. Fresh' and S. arcanum LA2172) and lowest in the most tolerant genotypes ( $S$. habrochaites LA2128 and S. arcanum LA2157) (Table 1). Spread of WT C. michiganensis to metaxylem vessels was significantly higher than $\triangle$ CmEXLX2 in $S$. lycopersicum 'Mt. Fresh' $(P=0.0012)$ and $S$. arcanum LA2172 $(P=0.0004)$, the two genotypes that had more severe symptoms with $\triangle$ CmEXLX2.

To achieve a closer view of biofilm-like structures in xylem vessels, we harvested transverse and longitudinal stem sections $1 \mathrm{~cm}$ above the inoculation site from plants $(12 \mathrm{dpi})$ inoculated with WT C. michiganensis for SEM. Bacterial aggregates were present in protoxylem vessels of all genotypes, and smaller aggregates were found less frequently in metaxylem vessels of $S$. lycopersicum 'Mt. Fresh', S. habrochaites LA2128, and S. arcanum LA2172 (Fig. 3) but not $S$. arcanum LA2157. C. michiganensis aggregates were present in xylem parenchyma and pith cells of S. lycopersicum 'Mt. Fresh' and S. habrochaites LA2128 (data not shown), but we cannot
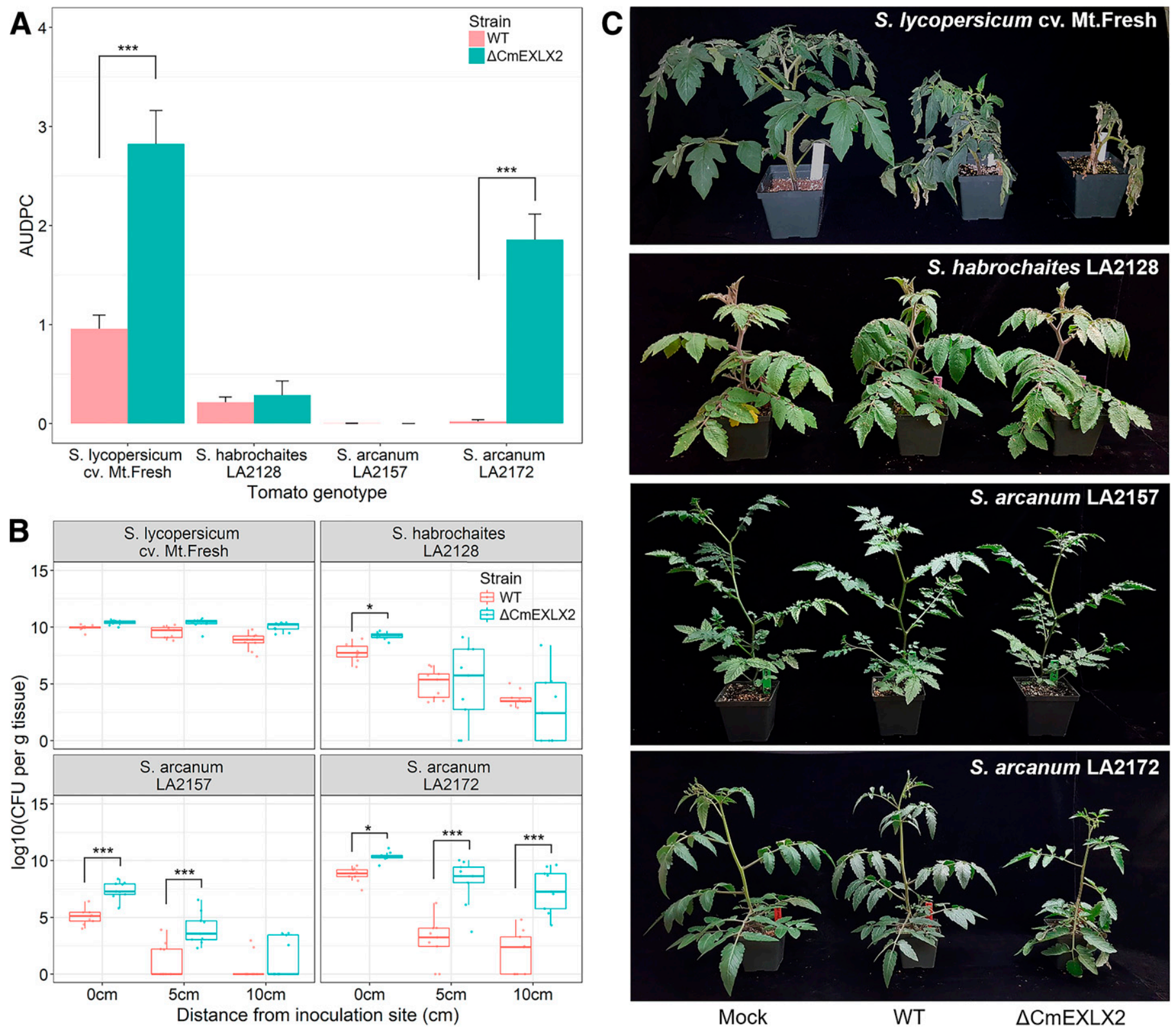

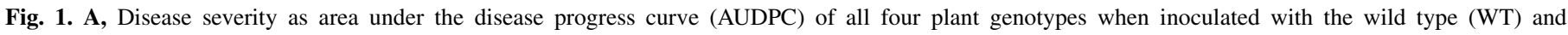

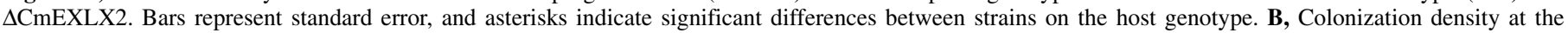

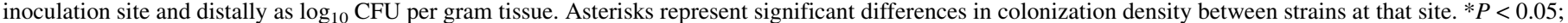
$* * * P<0.001$. C, Representative mock (left), WT (center), and $\Delta$ CmEXLX2-inoculated (right) plants at 21 days postinoculation. 
determine whether this was an artifact of sectioning or part of the natural infection process.

Fruit susceptibility assays demonstrate that susceptibility to $C$. michiganensis is tissue specific. The ability for $C$. michiganensis strains to produce bird's eye lesions on wild tomato fruit was tested between 7 and 9 dpa. Both WT and $\Delta$ CmEXLX2 caused characteristic symptoms, which appear as a necrotic center surrounded by a white halo, although the phenotype varied by plant genotype (Supplementary Fig. S6). C. michiganensis formed two- to threefold more lesions in S. lycopersicum 'Mt. Fresh' fruit in comparison with wild genotypes (Fig. 4C). Lesion severity was determined by measuring the diameter of whole lesions and their necrotic centers. Both WT and $\triangle \mathrm{CmEXLX} 2$ formed the largest lesions (approximately 1.3-mm diameter) on S. lycopersicum 'Mt. Fresh' and $S$. arcanum LA2157 fruit (Fig. 4A and Supplementary Figs. S6 and S7A). Lesion diameter of WT C. michiganensis was significantly larger $(P<0.05)$ in comparison with $\triangle$ CmEXLX2 lesions in S. habrochaites LA2128 and S. arcanum LA2172 fruit (Fig. 4A). $\triangle$ CmEXLX2 produced significantly larger necrotic lesions in S. lycopersicum 'Mt. Fresh' as has been previously reported (Tancos et al. 2018), but the size of the necrotic lesion was reduced relative to WT in S. habrochaites LA2128 and S. arcanum LA2172 (Fig. 4B).

Fruit from all four genotypes formed bird's eye lesions only when inoculated at early stages of development (7 to $15 \mathrm{dpa}$ ). Lesion phenotypes differed between genotypes, with the most striking difference being raised lesions in S. habrochaites LA2128 identified in $96 \%$ of lesions measured (Fig. 4D and Supplementary Fig. S6).

\section{DISCUSSION}

There are no commercially available sources of resistance to bacterial canker of tomato, and we are just beginning to understand the complex interactions between $C$. michiganensis and its hosts. Here, we examined colonization patterns of WT and a hypervirulent mutant $C$. michiganensis strain in xylem and fruit of tolerant wild tomato species. Previous studies characterizing wild tomato tolerance to $C$. michiganensis have inoculated older plants between 4- and 10-true leaf stages, which may prevent more severe symptoms from developing (Sen et al. 2012; Sharabani et al. 2013). To reduce the effect of plant age on assessing tolerance, we inoculated wild tomatoes seedlings during early development (twoto three-true leaf stage). Disease severity assays demonstrated that $\triangle \mathrm{CmEXLX} 2$ was hypervirulent in S. lycopersicum 'Mt. Fresh' and $S$. arcanum LA2172 but not in S. habrochaites LA2128 and $S$. arcanum LA2157. $\triangle \mathrm{CmEXLX2}$ colonized both $S$. arcanum accessions to higher densities than WT at several distances from the inoculation site, but stem cankers were only present in $S$. arcanum LA2172 inoculated with $\triangle \mathrm{CmEXLX} 2$. The lack of necrotic cankers
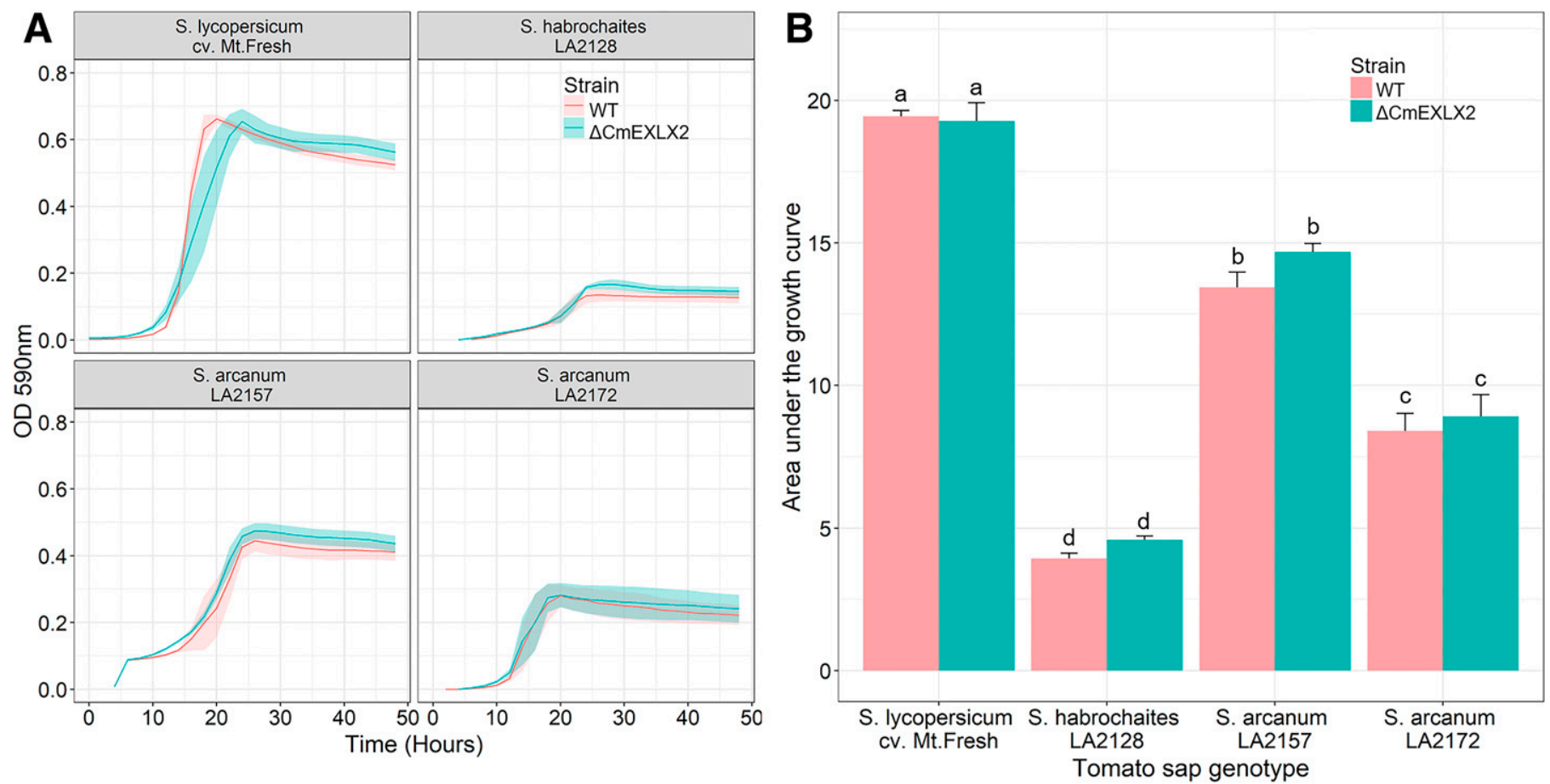

Fig. 2. A, Growth curves of the wild type (WT) and $\triangle$ CmEXLX2 cultured in sterile xylem sap collected from healthy plants measured by optical density (OD) at absorbance 590. Ribbon corresponds to $95 \%$ confidence intervals. B, Area under the growth curve of WT and $\Delta$ CmEXLX2 cultured in sterile xylem sap. Bars denote standard error, and letters represent significant differences $(P<0.05)$ between treatment groups using Tukey's pairwise comparisons.

TABLE 1. Percentage of protoxylem and metaxylem vessels infected by eGFP-expressing wild type (WT) and $\Delta$ CmEXLX2 Clavibacter michiganensis across four tomato genotypes determined using laser scanning confocal microscopy

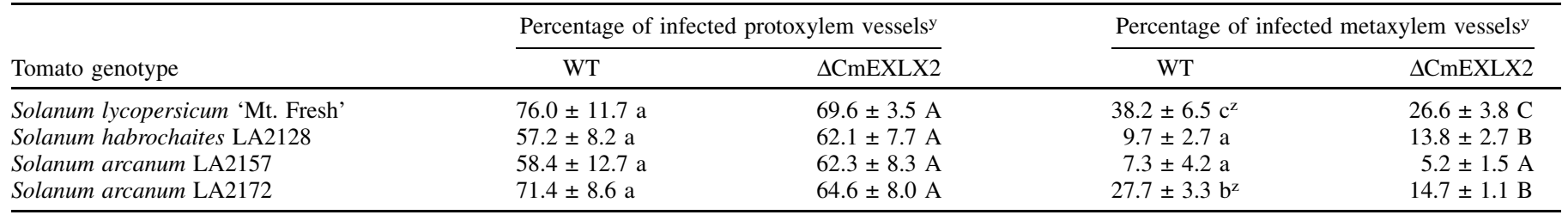

${ }^{y}$ Numbers presented are the mean percentage \pm standard deviation. Letters represent significant differences $(P<0.05)$ within each strain between plant genotypes. Lowercase letters represent differences or lack thereof between genotypes inoculated with WT. Uppercase letters denote differences or lack thereof between genotypes inoculated with $\triangle$ CmEXLX2.

z Significant differences $(P<0.05)$ between strains within a single plant genotype. 
in $S$. arcanum LA2157 and S. habrochaites LA2128 might suggest that $C$. michiganensis is impaired in its ability to macerate stem tissue in some wild tomato genotypes. We still do not understand the molecular mechanisms underlying hypervirulence, but these results suggest that secreted CmEXLX2 (Peritore-Galve et al. 2019) interacts with specific hosts, slowing disease onset and lowering severity of symptoms (Tancos et al. 2018). Functional analysis of a secreted Pectobacterium carotovorum expansin protein (PcEx11) determined that cellulolytic and xylanolytic activities of commercial cellulase and xylanase were reduced in celery vascular tissue in the presence of purified PcExl1 (Tovar-Herrera et al. 2018). Taken together, these results suggest that CmEXLX2 may bind plant polysaccharides to prevent hydrolysis. This may be a conserved strategy to slow disease progress or prevent activation of damageassociated molecular pattern-triggered immunity (Choi and Klessig 2016; Gust et al. 2017).

Previous studies of wild tomato tolerance have suggested that vascular morphology may play a role in tolerance to $C$. michiganensis (Coaker et al. 2002). A colonization study in cultivated tomato also demonstrated that $C$. michiganensis preferentially colonized protoxylem vessels during early infection and moved into metaxylem and parenchyma cells at later stages (Chalupowicz et al. 2012). Spread and colonization by WT and $\triangle \mathrm{CmEXLX} 2$ varied by host genotype. WT and $\triangle \mathrm{CmEXLX} 2$ colonized $S$. lycopersicum 'Mt. Fresh' to a high density at the inoculation site and distally, whereas colonization and spread were reduced in wild tomato genotypes. The association between $C$. michiganensis populations and symptom severity remains unclear. For example, the plasmid free derivative, $\mathrm{Cmm} 100$, is able to colonize and spread in S. lycopersicum to similar rates as the pathogenic strain Cmm382 (Meletzus et al. 1993), and other studies of wild tomato tolerance have shown that C. michiganensis can colonize stem tissue to high densities without causing severe symptoms (Francis et al. 2001; Sen et al. 2012). Assessment of systemic spread found that WT and $\triangle$ CmEXLX2 colonizes stem tissue to lower densities distally from the inoculation site in wild tomato species. We were unable to identify any tyloses or gels that may be obstructing the movement of $C$. michiganensis through the xylem through SEM and confocal microscopy; however, this does not rule out their presence.

Confocal microscopy revealed that both WT and $\triangle \mathrm{CmEXLX} 2$ were present mainly in protoxylem vessels of wild tomato plants during later stages of infection ( $21 \mathrm{dpi})$, which has been observed in S. lycopersicum and hypothesized as a strategy to keep its host alive longer (Chalupowicz et al. 2012). SEM images identified larger C. michiganensis aggregates in protoxylem vessels relative to metaxylem at 12 dpi for all genotypes except S. arcanum LA2157, where $C$. michiganensis was only found in protoxylem vessels. The ability for $C$. michiganensis to colonize higher proportions of metaxylem vessels correlated with disease severity between genotypes but not for differences between strains within a single genotype. A significantly higher proportion of metaxylem vessels was colonized by WT compared with $\triangle \mathrm{CmEXLX} 2$ in $S$. lycopersicum 'Mt. Fresh' and S. arcanum LA2172, which contradicts our hypothesis that metaxylem colonization is correlated to symptom severity. These data highlight the need for additional studies because the way in which $\triangle$ CmEXLX2 causes more severe symptoms than WT is unknown. Microscopy data suggest that $C$. michiganensis is impaired in its ability to spread through the vascular system of wild tomatoes, and it is possible that the restriction of $C$. michiganensis movement between xylem vessels and into other stem tissues may contribute to a reduction or elimination of symptoms in wild tomato species. However, further studies are required to delineate $C$. michiganensis colonization of xylem elements.

Biofilms play an important role in bacterial colonization and disease induction. Previous studies have shown that $C$. michiganensis is able to form biofilm-like aggregates in vitro when cultured in $S$. lycopersicum xylem sap but not in LB or minimal medium (Chalupowicz et al. 2012; Tancos et al. 2018). We hypothesized that wild tomato sap composition would hinder growth and aggregate formation in vitro. Xylem sap from wild tomato species sustained lower populations of $C$. michiganensis in vitro. In vivo bacterial populations did not correlate with growth in sap. For example, $C$. michiganensis grew to the lowest densities in $S$. arcanum LA2157 plants, but sap was able to sustain C. michiganensis growth at the second highest rate. The inverse was true in $S$. habrochaites LA2128, where sap was only able to sustain low levels of growth of $C$. michiganensis in vitro, but populations were only slightly reduced in planta in comparison with S. lycopersicum 'Mt. Fresh'. Contrasting the results of in vitro and in planta growth of $C$. michiganensis in wild tomato accessions suggests that host-C. michiganensis interactions are occurring in the xylem, affecting pathogen colonization. Aggregate formation was seemingly growth dependent, but attachment in vitro suggests that sap from wild tomato species provides similar conditions as sap from $S$. lycopersicum that triggers $C$. michiganensis attachment. We hypothesize that host proteins or metabolites are transported into xylem sap in response to $C$. michiganensis infection, which may lead to a reduction of bacterial growth as seen in S. arcanum LA2157. Metabolomics of $S$. lycopersicum xylem sap during infection by Ralstonia solanacearum found that the pathogen was secreting the polyamine putrescine as a virulence metabolite that altered host physiology (Lowe-Power et al. 2018). Findings in $R$. solanacearum support the idea that the xylem is an environment where pathogen and host interact to determine pathogen colonization.

Tomato fruit infection is an important disease stage for bacterial canker because it causes economic losses by blemishing fruit and can serve as a route for $C$. michiganensis infection of developing seed (Tancos et al. 2013). We tested if $C$. michiganensis would cause lesions on wild tomato fruit, and we found that wild tomatoes were susceptible to $C$. michiganensis infection and formed similar bird's eye lesions. Moreover, C. michiganensis was able to form bird's eye

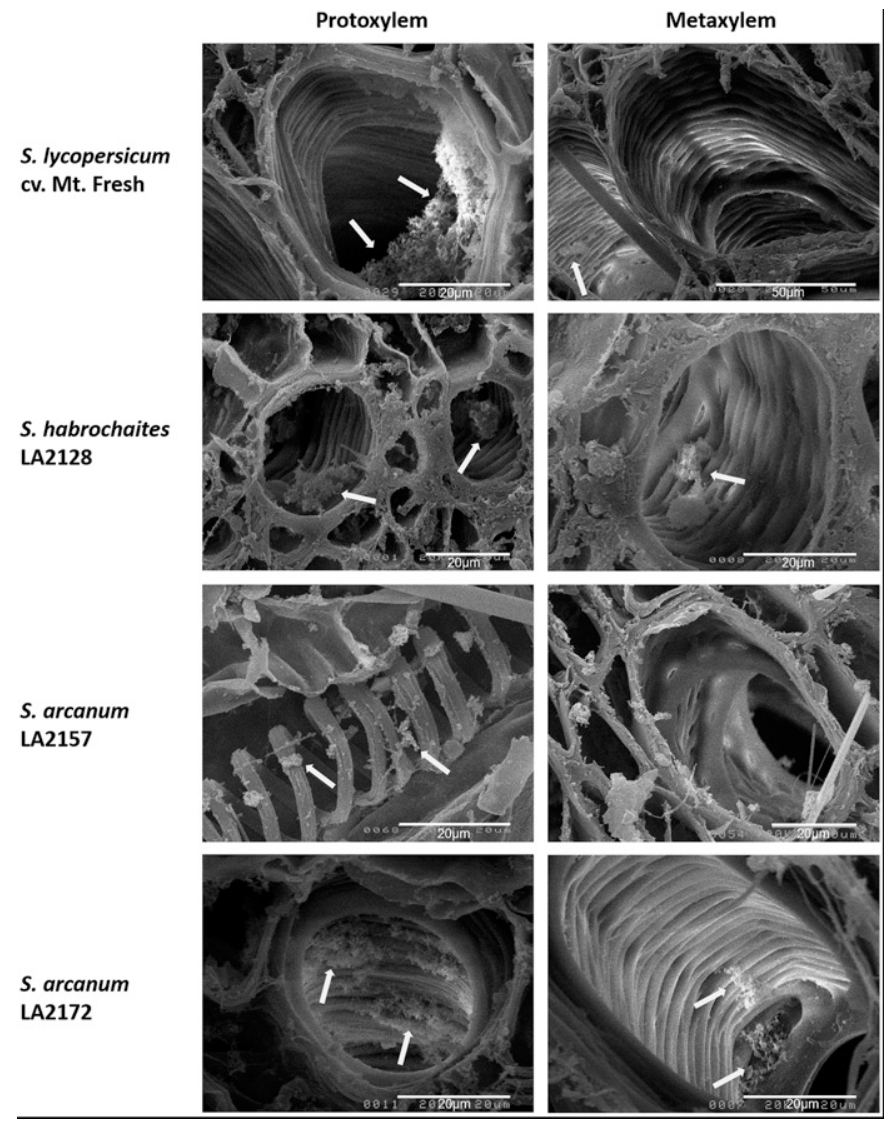

Fig. 3. Scanning electron microscope images of wild-type Clavibacter michiganensis aggregates in individual proto- and metaxylem vessels of all four plant genotypes. White arrows highlight bacterial aggregates. 
lesions on $S$. arcanum LA2157, which did not develop any symptoms when $C$. michiganensis was inoculated into the vascular system, demonstrating that $C$. michiganensis susceptibility can vary between fruit and vascular infections. Bird's eye lesions forming on fruit from all genotypes seem to suggest that there is a conserved tomato fruit response that causes the lesion phenotype. Interestingly, bird's eye lesions on S. habrochaites LA2128 manifested as raised bumps, which under a microscope, seemed to be caused by host cell proliferation at the infection site. Raised lesions that we are hypothesizing to be caused by host cell proliferation might be caused by perturbations in tomato fruit by compounds produced by C. michiganensis or through indirect alteration of host physiology as has been observed in other Gram-positive pathogens (Thapa et al. 2019).

In conclusion, our data provide evidence that $C$. michiganensis colonizes vascular and fruit tissues of wild tomato species but is reduced in systemic spread. C. michiganensis growth in sap and in planta was not correlated, suggesting that interactions between host and $C$. michiganensis impact pathogen growth in xylem vessels. Confocal microscopy was used to observe preferential protoxylem colonization and reduced spread to metaxylem in wild tomato genotypes, which may play a role in reducing disease severity. Finally, fruit inoculations revealed that asymptomatic responses to C. michiganensis can be specific to the inoculated tissue in wild
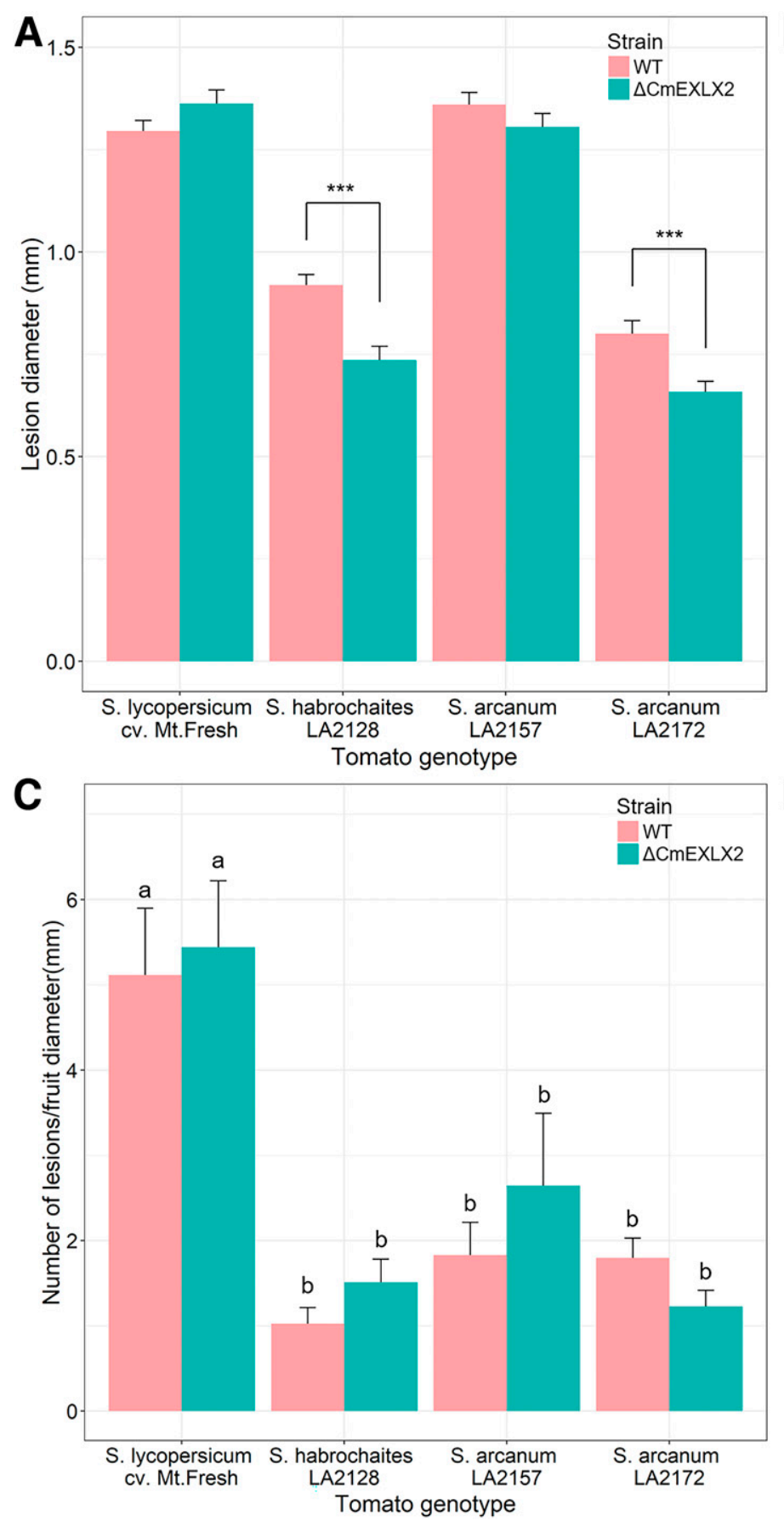

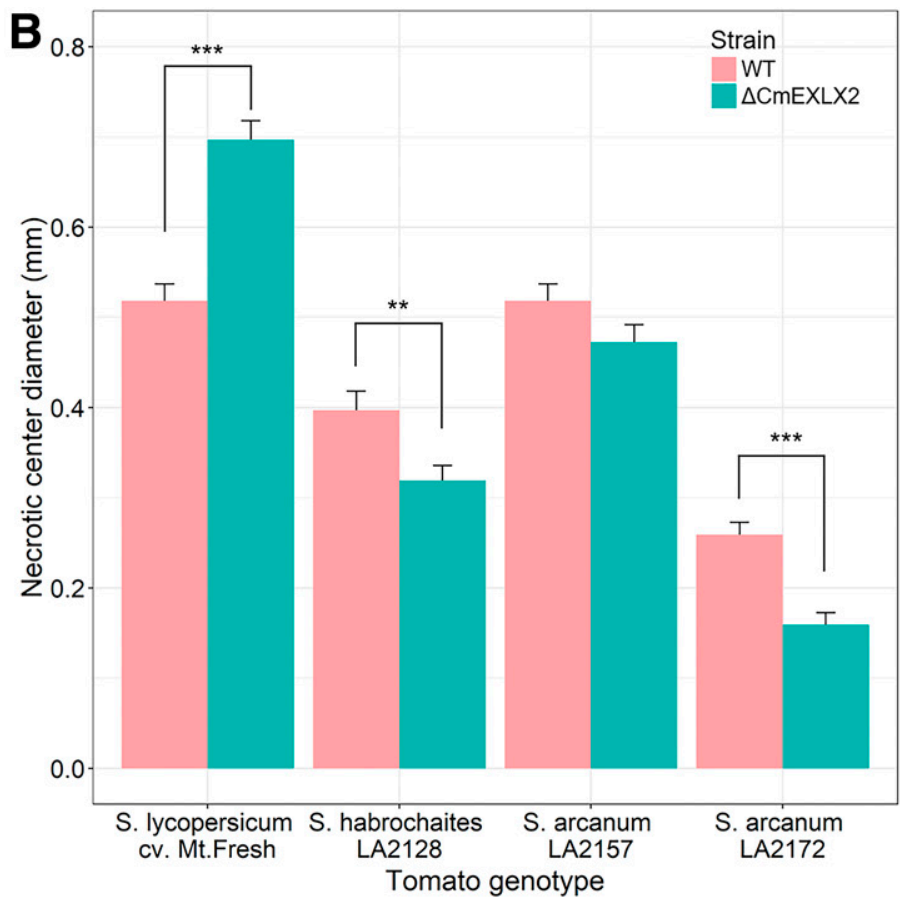

D
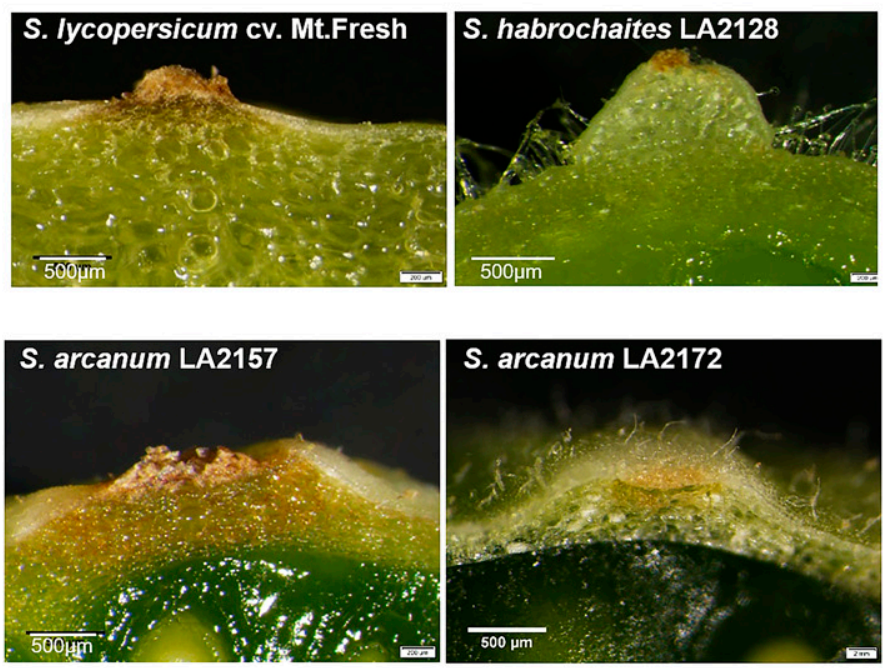

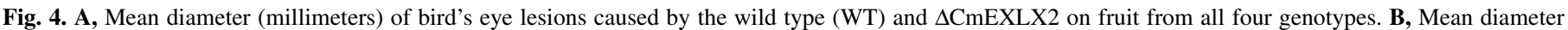

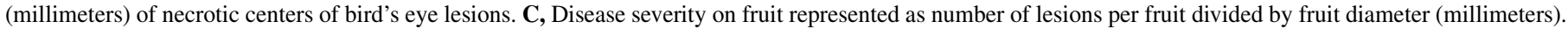

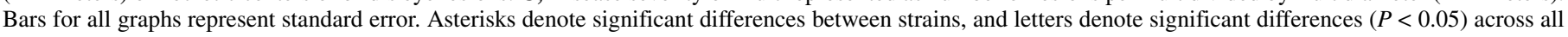

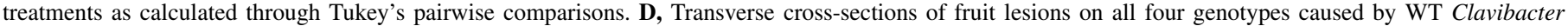
michiganensis demonstrating phenotypic differences of lesions. Scale bar is $500 \mu \mathrm{m} . * * P<0.01 ; * * * P<0.001$. 
tomato species. This is important to know because breeding efforts have focused largely on susceptibility of plants to $C$. michiganensis when inoculated into the vascular system but not onto fruit. Bacterial canker continues to be one of the largest threats to the tomato industry; therefore, understanding the pathology of this Gram-positive bacterium in tolerant and susceptible tomato genotypes can begin to untangle the complex interactions between host and pathogen.

\section{ACKNOWLEDGMENTS}

We thank the members of the laboratory of C. D. Smart, David Francis, and Angela Kruse for helpful discussions and critical feedback; Holly Lange, Taylere Herrmann, Juan Luis González Girón, Mariami Bekauri, and Mary Jean Welser for technical support; the Rick Tomato Genetics Resource Center and U.S. Department of Agriculture GRIN for wild tomato seed and advice on how to grow them; and Stephen Parry from the Cornell Statistical Consulting Unit for advice and support on statistical analyses.

\section{LITERATURE CITED}

Balaji, V., Mayrose, M., Sherf, O., Jacob-Hirsch, J., Eichenlaub, R., Iraki, N., et al. 2008. Tomato transcriptional changes in response to Clavibacter michiganensis subsp. michiganensis reveal a role for ethylene in disease development. Plant Physiol. 146:1797-1809.

Bates, D., Mächler, M., Bolker, B., and Walker, S. 2015. Fitting linear mixedeffects models using lme4. J. Stat. Softw. 67:1-48.

Chalupowicz, L., Barash, I., Reuven, M., Dror, O., Sharabani, G., Gartemann, K.-H., et al. 2017. Differential contribution of Clavibacter michiganensis ssp. michiganensis virulence factors to systemic and local infection in tomato. Mol. Plant Pathol. 18:336-346.

Chalupowicz, L., Zellermann, E.-M., Fluegel, M., Dror, O., Eichenlaub, R., Gartemann, K.-H., et al. 2012. Colonization and movement of GFP-labeled Clavibacter michiganensis subsp. michiganensis during tomato infection. Phytopathology 102:23-31.

Choi, H. W., and Klessig, D. F. 2016. DAMPs, MAMPs, and NAMPs in plant innate immunity. BMC Plant Biol. 16:232.

Coaker, G. L., and Francis, D. M. 2004. Mapping, genetic effects, and epistatic interaction of two bacterial canker resistance QTLs from Lycopersicon hirsutum. Theor. Appl. Genet. 108:1047-1055.

Coaker, G. L., Meulia, T., Kabelka, E. A., Jones, A. K., and Francis, D. M. 2002. A QTL controlling stem morphology and vascular development in Lycopersicon esculentum $\times$ Lycopersicon hirsutum (Solanaceae) crosses is located on chromosome 2. Am. J. Bot. 89:1859-1866.

Coaker, G. L., Willard, B., Kinter, M., Stockinger, E. J., and Francis, D. M. 2004. Proteomic analysis of resistance mediated by Rcm 2.0 and Rcm 5.1, two loci controlling resistance to bacterial canker of tomato. Mol. PlantMicrobe Interact. 17:1019-1028.

Davey, M. E., and O'toole, G. A. 2000. Microbial biofilms: From ecology to molecular genetics. Mol. Biol. Rep. 64:847-867.

de León, L., Siverio, F., Lopez, M. M., and Rodriguez, A. 2011. Clavibacter michiganensis subsp. michiganensis, a seedborne tomato pathogen: Healthy seeds are still the goal. Plant Dis. 95:1328-1338.

Dreier, J., Meletzus, D., and Eichenlaub, R. 1997. Characterization of the plasmid encoded virulence region pat-1 of phytopathogenic Clavibacter michiganensis subsp. michiganensis. Mol. Plant-Microbe Interact. 10: 195-206.

Francis, D. M., Kabelka, E., Bell, J., Franchino, B., and St. Clair, D. 2001. Resistance to bacterial canker in tomato (Lycopersicon hirsutum LA407) and its progeny derived from crosses to L. esculentum. Plant Dis. 85: 1171-1176.

Gartemann, K.-H., Abt, B., Bekel, T., Burger, A., Engemann, J., Flügel, M., et al. 2008. The genome sequence of the tomato-pathogenic actinomycete Clavibacter michiganensis subsp. michiganensis NCPPB382 reveals a large island involved in pathogenicity. J. Bacteriol. 190:2138-2149.

Gust, A. A., Pruitt, R., and Nürnberger, T. 2017. Sensing danger: Key to activating plant immunity. Trends Plant Sci. 22:779-791

Halekoh, U., and Højsgaard, S. 2014. A Kenward-Roger approximation and parametric bootstrap methods for tests in linear mixed models the $\mathrm{R}$ package pbkrtest. J. Stat. Softw. 59:1-32.

Jahr, H., Dreier, J., Meletzus, D., Bahro, R., and Eichenlaub, R. 2000. The endo- $\beta$-1,4-glucanase CelA of Clavibacter michiganensis subsp. michiganensis is a pathogenicity determinant required for induction of bacterial wilt of tomato. Mol. Plant-Microbe Interact. 13:703-714.

Kabelka, E., Franchino, B., and Francis, D. M. 2002. Two loci from Lycopersicon hirsutum LA407 confer resistance to strains of Clavibacter michiganensis subsp. michiganensis. Phytopathology 92:504-510.
Lara-Ávila, J. P., Isordia-Jasso, M. I., Castillo-Collazo, R., Simpson, J., and Alpuche-Solis, A. G. 2012. Gene expression analysis during interaction of tomato and related wild species with Clavibacter michiganensis subsp. michiganensis. Plant Mol. Biol. Report. 30:498-511.

Lenth, R. V. 2016. Least-squares means: The R package lsmeans. J. Stat. Softw. 69:1-33.

Li, X., Tambong, J., Yuan, K. (X.), Chen, W., Xu, H., Lévesque, C. A., and De Boer, S. H. 2018. Re-classification of Clavibacter michiganensis subspecies on the basis of whole-genome and multi-locus sequence analyses. Int. J. Syst. Evol. Microbiol. 68:234-240.

Lowe-Power, T. M., Hendrich, C. G., von Roepenack-Lahaye, E., Li, B., Wu, D., Mitra, R., et al. 2018. Metabolomics of tomato xylem sap during bacterial wilt reveals Ralstonia solanacearum produces abundant putrescine, a metabolite that accelerates wilt disease. Environ. Microbiol. 20:1330-1349.

Medina-Mora, C. M., Hausbeck, M. K., and Fulbright, D. W. 2001. Bird's eye lesions of tomato fruit produced by aerosol and direct application of Clavibacter michiganensis subsp. michiganensis. Plant Dis. 85:88-91.

Meletzus, D., Bermphol, A., Dreier, J., and Eichenlaub, R. 1993. Evidence for plasmid-encoded virulence factors in the phytopathogenic bacterium Clavibacter michiganensis subsp. michiganensis NCPPB382. J. Bacteriol. 175:2131-2136.

Mendiburu, F. D. 2015. Agricolae: Statistical Procedures for Agricultural Research. R Package. https://cran.r-project.org/web/packages/agricolae/index.html

Nandi, M., MacDonald, J., Liu, P., Weselowski, B., and Yuan, Z.-C. 2018. Clavibacter michiganensis ssp. michiganensis: Bacterial canker of tomato, molecular interactions and disease management. Mol. Plant Pathol. 19: 2036-2050.

Peritore-Galve, F. C., Schneider, D. J., Yang, Y., Thannhauser, T. W., Smart, C. D., and Stodghill, P. 2019. Proteome profile and genome refinement of the tomato-pathogenic bacterium Clavibacter michiganensis subsp. michiganensis. Proteomics 19:1800224.

Sen, Y., Feng, Z., Vandenbroucke, H., van der Wolf, J., Visser, R. G. F., and van Heusden, A. W. 2012. Screening for new sources of resistance to Clavibacter michiganensis subsp. michiganensis $(\mathrm{Cmm})$ in tomato. Euphytica 190:309-317.

Sen, Y., van der Wolf, J., Visser, R. G. F., and van Heusden, S. 2015. Bacterial canker of tomato: Current knowledge of detection, management, resistance, and interactions. Plant Dis. 99:4-13.

Sharabani, G., Shtienberg, D., Borenstein, M., Shulhani, R., Lofthouse, M., Sofer, M., et al. 2013. Effects of plant age on disease development and virulence of Clavibacter michiganensis subsp. michiganensis on tomato. Plant Pathol. 62:1114-1122.

Sotirova, V., Shtereva, L., Zagorska, N., Dimitrov, B., and Bogatsevska, N. 1999. Resistance responses of plants regenerated from tomato anther and somatic tissue cultures to Clavibacter michiganense subsp michiganense. Isr. J. Plant Sci. 47:237-243.

Tancos, M. A., Chalupowicz, L., Barash, I., Manulis-Sasson, S., and Smart, C. D. 2013. Tomato fruit and seed colonization by Clavibacter michiganensis subsp. michiganensis through external and internal routes. Appl. Environ. Microbiol. 79:6948-6957.

Tancos, M. A., Lange, H. W., and Smart, C. D. 2015. Characterizing the genetic diversity of the Clavibacter michiganensis subsp. michiganensis population in New York. Phytopathology 105:169-179.

Tancos, M. A., Lowe-Power, T. M., Peritore-Galve, F. C., Tran, T. M., Allen, C., and Smart, C. D. 2018. Plant-like bacterial expansins play contrasting roles in two tomato vascular pathogens. Mol. Plant Pathol. 19:1210-1221.

Thapa, S. P., Davis, E. W., Lyu, Q., Weisberg, A. J., Stevens, D. M., Clarke, C. R., et al. 2019. The evolution, ecology, and mechanisms of infection by Grampositive, plant-associated bacteria. Annu. Rev. Phytopathol. 57:341-365.

Thapa, S. P., Pattathil, S., Hahn, M. G., Jacques, M.-A., Gilbertson, R. L., and Coaker, G. 2017. Genomic analysis of Clavibacter michiganensis reveals insight into virulence strategies and genetic diversity of a Gram-positive bacterial pathogen. Mol. Plant-Microbe Interact. 30:786-802.

Tovar-Herrera, O. E., Rodríguez, M., Olarte-Lozano, M., Sampedro-Guerrero, J. A., Guerrero, A., Pinto-Cámara, R., et al. 2018. Analysis of the binding of expansin Ex11, from Pectobacterium carotovorum, to plant xylem and comparison to EXLX1 from Bacillus subtilis. ACS Omega 3:7008-7018.

Tran, T. M., MacIntyre, A., Hawes, M., and Allen, C. 2016. Escaping underground nets: Extracellular DNases degrade plant extracellular traps and contribute to virulence of the plant pathogenic bacterium Ralstonia solanacearum. PLoS Pathog 12:e1005686.

van Heusden, A. W., Koornneef, M., Voorrips, R. E., Brüggemann, W., Pet, G., Ginkel, R. V., et al. 1999. Three QTLs from Lycopersicon peruvianum confer a high level of resistance to Clavibacter michiganensis ssp. michiganensis. Theor. Appl. Genet. 99:1068-1074.

Vulkova, Z. V., and Sotirova, V. G. 1993. Study of the three-genome hybrid Lycopersicon esculentum Mill. —L. chilense Dun. — L. peruvianum var 'humifusum' Mill. and its use as a source for resistance. Theor. Appl. Genet. 87:337-342. 